Hypoxia characterizes growing tumors and contributes significantly to their aggressiveness. Hypoxia-inducible factors (HIFs 1 and 2) are stabilized and act differentially as transcription factors on tumor growth and are responsible for important cancer hallmarks such as pathologic angiogenesis, cellular proliferation, apoptosis, differentiation and genetic instability as well as affecting tumor metabolism, tumor immune responses, invasion and metastasis. Taking into account the tumor tissue as a whole and considering the interplay of the various partners which react with hypoxia in the tumor site lead to reconsideration of the treatment strategies. Key limitations of treatment success result from the adaptation to the hypoxic milieu sustained by tumor anarchic angiogenesis. This raises immune tolerance by influencing the recruitment of immunosuppressive cells as bone marrow derived suppressor cells (MDSC) or by impairing the infiltration and killing of tumor cells by cytotoxic cells at the level of the endothelial cell wall of the hypoxic tumor vessels, as summarized in the schematic abstract.

Key words: angiogenesis, hypoxia, immunosuppression, microenvironment.

Contemp Oncol (Pozn) 2018; 22 (1A): 7-13 DOI: https://doi.org/10.5114/wo.2018.73874

\section{The role of hypoxia in shaping the recruitment of proangiogenic and immunosuppressive cells in the tumor microenvironment}

\author{
Salem Chouaib ${ }^{1,2,3}$, Viktor Umansky ${ }^{4,5}$, Claudine Kieda $a^{6,7}$ \\ ${ }^{1}$ INSERM (Institut National de la Santé et de la Recherche Médicale) UMR1186, \\ Laboratory Integrative Tumor Immunology and Genetic Oncology, Villejuif, France \\ ${ }^{2}$ INSERM, Gustave Roussy, Univ. Paris-Sud, Universite Paris-Saclay, Villejuif, France \\ ${ }^{3}$ Thumbay Institute for Precision Medicine and Translational Research - Gulf Medical \\ University Ajman UAE \\ ${ }^{4}$ Skin Cancer Unit, German Cancer Research Center (DKFZ), Heidelberg, Germany \\ ${ }^{5}$ Department of Dermatology, Venereology and Allergology, University Medical Center \\ Mannheim, Ruprecht-Karl University of Heidelberg, Mannheim, Germany \\ ${ }^{6}$ Laboratory of Molecular oncology, Military Institute of Medicine, Warsaw, Poland \\ ${ }^{7}$ Centre for Molecular Biophysics, CNRS, 45071, Orleans, France
}

\section{Microenvironmental hypoxia in shaping the angiogenic reaction controls tumor immune resistance}

During tumor growth, endothelial cells first react to hypoxia. The early signals that reach the endothelial cells are produced as a response of the cancer cells to the hypoxic stress to which they are submitted while they develop. The hypoxia-mediated response of tumor cells to such lack of oxygen resulted in the stabilization of the transcription factor HIF-1 and the transcription cascade that starts upon binding of the heterodimer HIF- $1 \alpha \beta$ to the hypoxia response element (HRE) [1]. The synthesis of the main factors which turn on angiogenesis is induced to fight the hypoxic conditions inside the tumor site. Indeed, angiogenesis is the only mechanism by which the blood can be introduced into the tumor site to bring nutriments and oxygen to the tumor cells and allow tumor progression [2]. It is, by now, clear that tumor angiogenesis is pathologic and cannot establish efficient blood flow inside the tumor [3]. This, consequently, maintains chronic hypoxia and a permanent proangiogenic state characteristic of the tumor microenvironment (TME). According to the antiangiogenesis-based approaches long used for tumor treatment, angiogenesis should be totally destroyed to starve and exhaust the tumor [4].

This strategy could not be applied as the Warburg effect brings the means for the tumor cells to overtake the lack of nutriment to produce energy by the process of anaerobic glycolysis [5]. The tumor cells set their rescue mechanism through the induction of glucose receptors which start the glycolysis cascade [6]. This energetic rescue metabolism uses the glucose-containing substrates in the tumor milieu and glucosamine/ $\mathrm{N}$-acetyl glucosamine pathway leading to the production of lactate and the reduction of $\mathrm{pH}$ of the tumor microenvironment [7].

The second reason why such an antiangiogenic strategy could not be applied results from the similarities in endpoints of both the pathologic angiogenesis and the antiangiogenic treatments, the second being the limit of the first. The antiangiogenic treatment effect pushes forward the rescue mechanisms that the cells have to raise in order to survive.

In such conditions, the antiangiogenic treatments have been shown to favor the selection of resistant tumor cells [5]. They are dedifferentiated and, in a quiescent state, remain able to survive in very hypoxic and acidic conditions [8]. We have directly demonstrated that not only are these cells 
selected and their phenotype maintained by hypoxia, but they are also, in the tumor, responsible for the selective recruitment of endothelial progenitor cells as opposed to mature endothelial cells [9]. Such stem-like cells are prone to produce the whole tumor repertoire as soon as the microenvironment conditions allow it. They are turned on for differentiation and growth by cooperation with given stromal cells such as endothelial progenitor cells (EPCS) and carcinoma-associated fibroblasts (CAFS) $[9,10]$.

Similarly to tumor cells, endothelial cells use the glycolytic mechanism for growth even in normal, non-hypoxic, conditions [11]. In the tumor but also normoxic (physiological value of oxygen in tissues) [12] conditions the endothelial cells use the glucose and glucosamine degradation pathways to perform angiogenesis in response to stimulatory mechanisms [13]. The paracrine effect of proangiogenic molecules produced by the tumor cells such as VEGF-A, -B, -C, IL-8, PDGF, and angiopoietins 1 and 2, in response to hypoxia join, during endothelial cell angiogenic process, the hypoxic response and the glycolytic degradation process [14].

Endothelial cells coordinate the proper expansion of the vessels through the NOTCH/DLL4 mechanism of stalk/ tip signaling [15]. Notch is controls activation of the tumor suppressor PTEN in the endothelial cells [16]. In the absence of PTEN phosphatase activity, angiogenic growth is constantly stimulated because of the constant PI3K/AKT/ mTOR active pathway which results in anarchic tumor angiogenesis [17].

Consequently, the initial angiogenic switch coming from the hypoxic tumor cells is addressed to the non-hypoxic endothelial cells and recruits them to form vessels inside the tumor by the chemotactic effect of hypoxia response molecules such as VEGF-A and IL-8. Endothelial cells are thus recruited into the tumor by a non-hypoxia-dependent mechanism from the preexisting vessels close to the tumor and the bone marrow from where the endothelial progenitors are mobilized $[15,18]$.

The endothelial cells' response to the tumor proangiogenic switch is first indirectly related to hypoxia. In a second step, the process of tumor vessel formation occurs and is maintained by the hypoxic microenvironment [19]. In these conditions the endothelial reaction to produce the tumor induced angiogenesis is totally regulated and dependent on the constancy of the hypoxia parameter. We have shown that PTEN activation in endothelial cells is directly related to hypoxia reoxygenation and the maintenance of PTEN activation is a key condition which allows vessel changes from pathologic to stably normalized [19]. The importance of angiogenesis normalization was later confirmed, inhibiting the glycolytic activity of the endothelial metabolism, leading to the same anti-metastatic effects [20].

At the endothelial cell level normalization translates into a series of rearrangements and differential expression of antigens as well as secreted molecules playing a strong role in the immunomodulation. Under hypoxic conditions the molecules expressed on the endothelial cell surface participate in the intercellular recognition with cancer cells as well as with immune cells. As such the endothelial barri- er not only helps the intravasation and further dispersion of metastatic cells but also modulates the adhesion properties and extravasation of immunocompetent cells and/ or immunosuppressive cells. The resulting cellular and molecular composition of the tumor microenvironment allows a relative classification of cancers for further personalized immunotherapeutic protocols [21].

The endothelial reaction in hypoxia contributes to selection, differently than in normoxia, of the immune cells' entry inside the tumor. We could show that although the direct interaction of NK cells with endothelial cells [22] is increased upon NK cell activation by IL-2 [23] as well as IL-15 and IL-27 [24], hypoxia modulates the recognitions in an opposite manner by reducing NK cell adhesion to the tumor endothelium in the mammary carcinoma [10], similarly to the case of multiple myeloma-associated endothelial cells [24].

Contributing to the hypoxia-dependent immunosuppressive effect of tumor angiogenesis, the expression of soluble molecules such as VEGFs exerts a strong chemoattraction towards efficient suppressive cells such as MDSCs, as described in detail below. Immune cells can express VEGF receptors as CD4 $4^{+}$forkhead box protein $\mathrm{P3}$ $($ FOXP3)+ regulatory $T$ cells which are chemoattracted by VEGF inside the tumors, where they suppress the anti-tumor immune response by CTLS and NKs [25]. Vessels normalization reduces it [18]. Linked to this, endothelial cells in the tumor are ruled for their immunosuppressive effect to the reduction of PTEN activation. The tumor suppressor effect indeed controls PI3K activation, which activates the mammalian target of rapamycin (mTOR), which is stimulated in a hypoxic and nutrient-poor environment. PTEN antagonizes the PI3 kinase pathway mainly, but also has phosphatase-independent functions. In cancer, the PTEN status determines the response to chemotherapy through the normalization effect of vessels on endothelial cells, highlighting the advantage of monitoring PTEN expression and activity and developing PTEN-targeted therapies. PTEN inactivation by hypoxia has a direct immunosuppressive effect by the expression of immune checkpoint molecules as PD-L1 and PD-L2 on the endothelial cell surface [26-29]. Activity of immune checkpoints' PD-L1 expression is directly involved in the reduced activity of NK cells which as T cells are PD1+ [30] and PD-L1 expression and the immunosuppressive effect is under the control of PTEN [31].

Therefore, endothelial cells in the hypoxic tumor site establish a physical barrier that prevents homing of effective tumor-rejecting cells such as NK cells and CTLS, and suppresses effector lymphocytes through the recruitment and activation of immunosuppressive cells such as myeloid-derived suppressor cells, tolerogenic monocytes and $T$ regulatory cells. The anti-tumor strategies which aim at endothelial PTEN as a molecular target may open new adjuvant approaches for immunotherapy.

\section{Role of microenvironmental hypoxia in shaping tumor MDSC recruitment and immunosuppression}

It has been well documented in the recent decade that various tumors were able to develop immune es- 
cape mechanisms dealing with structural and functional changes both in tumor and stroma cells, leading finally to the inability of even activated effector immune cells to reject the tumor [32]. These mechanisms were reported to include i) alterations of MHC class I expression and components of the antigen processing machinery [33, 34], ii) intensive, long-term secretion of inflammatory and immunosuppressive factors such as vascular endothelial growth factor (VEGF), transforming growth factor (TGF)- $\beta$, interleukin (IL)-1 $1 \beta$, IL-6, IL-10, granulocyte-macrophage colony-stimulating factor (GM-CSF), reactive oxygen and nitrogen species or prostaglandins $[35,36]$, and iii) hypoxic conditions inducing the expression of transcription factor hypoxia inducible factor (HIF)-1 $\alpha$ [37]. In this regard, tumor cells were found to express HIF-1 $\alpha$ released chemokines such as CCL2, CCL3, C-X-C chemokine receptor (CXCR)-4, CCL5, CCL7, CCL8, CCL9, and CXCL12, as well as growth factors such as CSF-1, macrophage stimulating protein (MSP), GM-CSF, semaphorin $3 \mathrm{~A}$ and TGF- $\beta$, that will help attract monocytes from the circulation [37, 38].

It has been demonstrated in numerous reports that myeloid-derived suppressor cells (MDSC) could play a critical role in the development of the immunosuppressive tumor microenvironment [35, 39-42]. This extremely heterogeneous population of immature myeloid cells representing precursors of granulocytes, macrophages, and DCs has recently attracted much attention as one of the key cells promoting tumor progression and creating the immunosuppressive tumor microenvironment. Importantly, MDSC acquire strong immunosuppressive functions that allow them to efficiently inhibit T-cell mediated anti-tumor reactivity by various mechanisms [35, 40, 41, 43]. In mice, MDSCs express Gr1 and CD11b surface molecules and consist of two major subsets: polymorphonuclear CD11b+Ly6G+Ly6Clo (PMN-MDSCS) and monocytic CD11b+Ly6G-Ly6Chi (M-MDSCs) [40, 44, 45]. In humans, MDSCs are characterized as Lin HLA-DR-/10 $\mathrm{CD}^{-1} 3^{+}$or Lin $\mathrm{HLA}^{-\mathrm{DR}}{ }^{-1}$ ${ }^{10} \mathrm{CD} 11 b^{+} \mathrm{CD} 14 \mathrm{CD}^{+} 5^{+} \mathrm{CD} 33^{+}$for PMN-MDSCs and $\mathrm{CD}^{+} 4^{+} \mathrm{H}-$ LA-DR ${ }^{\text {neg/lo }}$ or Lin ${ }^{-H L A}-D R^{\text {neg/lo }} \mathrm{CD} 11 b^{+} \mathrm{CD} 14^{+} \mathrm{CD} 15$ for M-MDSCs [45-47]. MDSCs have been shown to derive from bone marrow hematopoietic precursors due to the alteration of myelopoiesis by chronic inflammatory mediators such as granulocyte-macrophage colony-stimulating factor (GMCSF), granulocyte colony-stimulating factor (G-CSF), macrophage colony-stimulating factor (M-CSF), stem cell factor (SCF), VEGF, IL-6, IL-1 $\beta$ as well as chemokines CCL2, CCL3, CCL4, CCL5 etc. [35, 36, 40, 42, 48, 49]. These factors are known to be produced both by tumor and stroma cells, and the signaling involves mainly the signal transducer and activator of transcription 3 (STAT3), preventing MDSC differentiation and promoting their proliferation [50, 51]. However, this is not sufficient for the induction of MDSC immunosuppressive activity that is provided by pro-inflammatory molecules such as interferon (IFN)- $\gamma$, IL-1 $\beta$, IL-4, IL-13, TNF- $\alpha$, toll-like receptor (TLR) ligands, and prostaglandin (PGE) E2 and is mediated by STAT1, STAT6 and nuclear factor (NF)- $\mathrm{KB}$ transcription factors as well as by cyclooxygenase (COX)-2 upregulation [35, 36, 43, 50, 52].

Studies published in recent years demonstrated that the generation, enrichment and activation of MDSCs could be mediated not only by chronic exposure to inflammatory factors but also by hypoxia typical for the tumor microenvironment $[53,54]$. It was found that HIF-1 $\alpha$ induced in the tumor microenvironment under hypoxia conditions could strongly up-regulate expression of inducible nitric oxide (NO) synthase and arginase (ARG)-1 [55, 56]. NO has been found to induce $T$ cell apoptosis as well as the nitration of chemokines and T cell receptors (TCR) that block $T$ cell migration and cytotoxic effects against tumor cells; finally, these molecules could inhibit production of cytokines (such as IL-2), which are crucial for T cell anti-tumor functions [35, 41, 43, 57-59]. ARG-1 activation results in the deprivation of arginine, which is important for multiple $T$ cell functions $[35,39,60]$. In particular, the lack of arginine induces the down-regulation of TCR $\zeta$-chain expression, blocking the transmission of activation signals from the cell membrane $[42,43,48]$. Such hypoxia-related upregulation of $\mathrm{NO}$ production and $\mathrm{ASRG}-1$ expression was reported to be mediated by the binding of HIF-1 $\alpha$. Such hypoxia-related upregulation of NO production and ASRG1 expression was reported to be mediat [56]. Interestingly, miR-210 overexpression was sufficient to enhance MDSC-mediated T-cell suppression under normoxic conditions, while targeting hypoxia-induced miR-210 was sufficient to decrease MDSC function against T cells [56]. In addition to the ability to suppress $T$ cell functions, hypoxia promotes differentiation of MDSC to immunosuppressive tumor-associated macrophages, further supporting the immunosuppressive network in the tumor microenvironment [55].

Recently, it has been demonstrated that HIF-1 $\alpha$ (but not HIF-2 $\alpha$ ) could also strongly upregulate expression of the negative immune checkpoint molecule programmed death ligand (PD-L)-1 (also known as B7-H1 or CD274 on MDSC from tumor bearing mice [61]. The interaction between PD-L1 and its receptor PD-1 originally discovered as a protein involved in programmed cell death [62] and expressed on activated T cells [63] has been reported to downregulate the anti-tumor reactivity of $T$ cells infiltrating tumor lesions $[64,65]$. Importantly, PD-L1 expression was significantly increased not only on MDSC [63] but also on macrophages, dendritic cells, and tumor cells via direct binding of HIF-1 $\alpha$ to a hypoxia-response element in the PD-L1 proximal promoter [61].

Hypoxic tumor cells were demonstrated to secrete various soluble factors that could condition pre-metastatic niches by recruiting CD11b+/Ly6C $\mathrm{Cmed}^{\mathrm{L}} \mathrm{Ly} 6 \mathrm{G}^{+}$polymorphonuclear MDSC that inhibited NK cell functions [66]. A more hypoxic tumor microenvironment induced by tumor pericyte abnormalities leading to defective tumor vasculature has been found to increase the recruitment of MDSC to the tumor site [67]. Another mechanism of MDSC recruitment to the tumor microenvironment stimulated by HIF-1 $\alpha$ and HIF- $2 \alpha$ includes activation of chemokine (C-C motif) ligand $(\mathrm{CCL}) 26$ production in cancer cells interacting with its receptor CXCR1 on MDSC [68]. Knockdown of CCL26 in cancer cells, and inhibition of its production by the HIF inhibitor or blockade of CXCR1 with respective neutralizing antibodies, could substantially suppress MDSC recruitment angiogenesis, and tumor growth in MDSC [68]. 
Hypoxia has also been reported to induce in the tumor microenvironment production of adenosine, which is known to inhibit T cell anti-tumor functions via interaction with $\mathrm{A} 2$ adenosine receptors on the surface of effector $T$ cells [69-72]. Adenosine accumulation outside the cell has been reported to be induced by increased expression of ectonucleotidases CD73 and CD39 [73, 74]. Although the activation of these enzymes was attributed initially to tumor cells and regulatory $T$ cells $[71,75,76]$, tumor infiltrating MDSC have been recently demonstrated to upregulate the expression of CD73, leading to elevated adenosine production and thereby to immunosuppression [72]. Moreover, it has been found that extracellular adenosine induced MDSC expansion and stimulation through the receptor A2B [77]. High CD73 levels were detected on the surface of PMN-MDSC, and the substrate of CD73, adenosine monophosphate, could elevate immunosuppressive activity of MDSC in vitro $[72,78]$. Importantly, the observed upregulation of CD73 on tumor-infiltrating MDSC could be induced in a HIF-1 $\alpha$-dependent manner similar to such upregulation reported for regulatory T cells [79].

Taken together, hypoxic conditions typical for the tumor microenvironment could not only induce the trafficking of MDSC into the tumor site but also stimulate their capacities to inhibit anti-tumor reactivity of T and NK cells by various mechanisms.

\section{Role of microenvironmental hypoxia in shaping immune reaction and tumor resistance to killer cells}

Hypoxia - a key determinant

of microenvironment hostility

The crosstalk between stromal cells and malignant cells within the tumor microenvironment crucially determines the fate of tumor progression, its hostility and heterogeneity. It is well established that the tumor microenvironment (TME) supports tumor growth and limits the effectiveness of solid tumor immunotherapies by promoting neoplastic transformation and cell plasticity and by inducing tumor cell resistance to host immunity. Accumulating data suggest that hypoxic stress in the TME promotes several tu- mor escape mechanisms, including immune suppression and the emergence of tumor-resistant variants. Highly aggressive and rapidly growing tumors are exposed to hypoxia or even anoxia that occurs as a consequence of inadequate and/or irregular blood supply. It is widely reported that gradients of hypoxia occur in most solid tumors and cells found in hypoxic regions are associated with the most aggressive and therapy-resistant fractions of the tumor. Under hypoxia or pseudohypoxia, cells activate a number of adaptive responses coordinated by various cellular pathways. Despite the ubiquity and importance of hypoxia responses, little is known about the impact of hypoxic stress on the anti-tumor cytotoxic immune response.

Hypoxia induces resistance to cell-mediated cytotoxicity

Although the advent of new immunotherapeutic approaches has improved the survival of many patients with advanced malignancies, the high prevalence of non-responders also provides a strong reminder that we possess only a partial understanding of the events underlying the immune resistance of tumors. The ultimate goal of most cancer immunotherapy strategies is to induce a strong cytotoxic $T$ lymphocyte $(\mathrm{CTL})$ response. The prevailing view is that the generation of a sufficiently high frequency of $C T L$ response will result in tumor regression. However, it has become increasingly apparent in both preclinical models and patient trials that tumors can efficiently evade or inactivate even substantial immune responses by denying $T$ cells access to the tumor, by establishing a metabolically hostile microenvironment, and through selection of immune-resistant tumor cell variants. In this regard, we showed that hypoxia induced tumor resistance to CTL-induced killing [80]. We next provided evidence indicating that hypoxia-induced autophagy impairs CTL-mediated tumor cell lysis by regulating phospho-STAT3 in target cells [81]. Additionally, boosting the CTL response, using a TRP2-peptide vaccination strategy, and targeting autophagy in hypoxic tumors improves the efficacy of this cancer vaccine and promotes tumor regression in vivo [81]. More recently, we have reported that attenuation of miR-210 in hypoxic

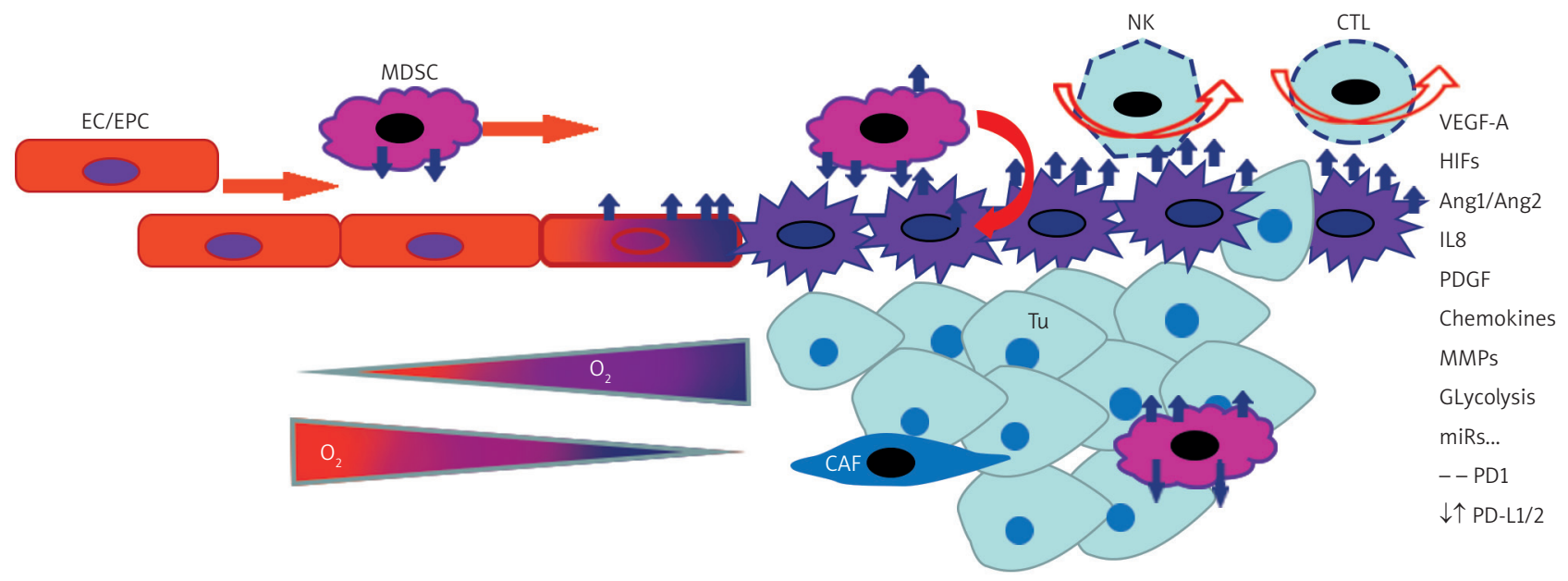

Fig. 1. Hypoxia vs normoxia in the tumor microenvironment balancing the immunosuppressive cells and cytokine production 
cells can significantly restore susceptibility to autologous CTL-mediated lysis, independent of tumor cell recognition and $\mathrm{CTL}$ reactivity [82]. A comprehensive approach using transcriptome analysis, Argonaute protein immunoprecipitation and a luciferase reporter assay revealed that the genes PTPN1, HOXA1 and TP53/11 were miR-210 target genes regulated in hypoxic cells. Further analysis showed that silencing of PTPN1, HOXA1 and TP53/11 dramatically decreased tumor cell susceptibility to CTL-mediated lysis [82]. We have also demonstrated that hypoxic tumor cells can escape NK-mediated immune surveillance by activating autophagy under hypoxia [83, 84]. Janji et al. [83, 84] have shown that Granzyme B is selectively degraded upon activation of autophagy in hypoxic cells, thereby inhibiting NK-mediated target cell apoptosis [84]. More recently, the role of autophagy in regulating NK-mediated immune responses was investigated in a clear cell renal cell carcinoma cell model harboring mutation in the VHL gene and known to be resistant to NK-mediated killing [85].

\section{Conclusions}

The novel immunotherapeutic approaches have provided durable remission in a significant number of cancer patients with cancers previously considered rapidly lethal. Nonetheless, the high degree of non-responders, and in some cases the emergence of resistance in patients who do initially respond, represents a significant challenge in the field of cancer biology and immunotherapy.

Besides the several mechanisms known to alter the immune responses to tumors, the presence of intratumoral hypoxia is significantly associated with increased risk of treatment failure, invasion, metastasis, and patient mortality. This is, in part, due to inappropriate local immune reaction and resistance of hypoxic tumor cells to cytotoxic treatments including immune cell-mediated cytotoxicity.

Since intratumoral hypoxia has long been considered as a driving force of tumor progression and to play a key role in remodeling the tumor stroma and favoring the emergence of tolerance, immune suppression and tumor resistance, efforts to incorporate components of the hypoxic microenvironment are necessary to guide the successful design of future cancer immunotherapeutic approaches. Targeting the hypoxic tumor microenvironment to awaken or reawaken immune cells, or to redirect it from a pro-tumor to an anti-tumor state, is at present a pertinent strategy.

This work was supported by grants from Ligue Contre le Cancer (EL2015.LNCC/SaC), Institut National du Cancer (PLBIO15-266), and the SIRIC-SOCRATE program; and from the Polish Ministry of Defence grant Kosciuszko I no. 579_2016_DA.

\section{References}

1. Semenza GL. Targeting HIF-1 for cancer therapy. Nat Rev Cancer 2003; 3: 721-32.

2. Semenza GL. HIF-1 and mechanisms of hypoxia sensing. Curr Opin Cell Biol 2001; 13: 167-71.
3. Kerbel RS. Tumor angiogenesis. N Engl J Med 2008; 358: 2039-49.

4. Koh YJ, Kim HZ, Hwang SI, et al. Double antiangiogenic protein, DAAP, targeting VEGF-A and angiopoietins in tumor angiogenesis, metastasis, and vascular leakage. Cancer Cell 2010; 18: 171-84.

5. Pàez-Ribes M, Allen E, Hudock J, et al. Antiangiogenic therapy elicits malignant progression of tumors to increased local invasion and distant metastasis. Cancer Cell 2009; 15: 220-31.

6. Jain RK. Antiangiogenesis Strategies Revisited: From Starving Tumors to Alleviating Hypoxia. Cancer Cell 2014; 26: 605-22.

7. Polet F, Feron O.Endothelial cell metabolism and tumour angiogenesis: glucose and glutamine as essential fuels and lactate as the driving force. J Intern Med 2013; 273: 156-65.

8. Grau JJ, Mesía R, de la Iglesia-Vicente M, et al. Enrichment of cells with cancer stem cell-like markers in relapses of chemoresistant patients with locally advanced head and neck squamous cell carcinoma. Oncology 2016; 90: 267-72.

9. Klimkiewicz K, Weglarczyk K, Collet G, et al. A 3D model of tumour angiogenic microenvironment to monitor hypoxia effects on cell interactions and cancer stem cell selection. Cancer Lett 2017; 396: 10-20.

10. Tejchman A, Lamerant-Fayel N, Jacquinet JC, et al. Tumor hypoxia modulates podoplanin/CCL21 interactions in CCR7+ NK cell recruitment and CCR7+ tumor cell mobilization. Oncotarget 2017; 8: 31876-87.

11. Sodi VL, Khaku S, Krutilina R, et al. mTOR/MYC Axis Regulates O-GlcNAc Transferase Expression and O-GlcNAcylation in Breast Cancer. Mol Cancer Res 2015; 13: 923-33.

12. Carreau A, El Hafny-Rahbi B, Matejuk A, Grillon C, Kieda C. Why is the partial oxygen pressure of human tissues a crucial parameter? Small molecules and hypoxia. J Cell Mol Med 2011; 15: 1239-53.

13. De Bock K, Georgiadou M, Schoors S,et al. Role of PFKFB3-driven glycolysis in vessel sprouting. Cell 2013; 154: 651-63.

14. De Bock K, Georgiadou M, Carmeliet P. Role of endothelial cell metabolism in vessel sprouting. Cell Metab 2013; 18: 634-47.

15. Blanco R, Gerhardt H. VEGF and Notch in tip and stalk cell selection. Cold Spring Harb Perspect Med 2013; 3: a006569.

16. Serra H, Chivite I, Angulo-Urarte A, et al. PTEN mediates Notch-dependent stalk cell arrest in angiogenesis. Nat Commun 2015; 6: 7935.

17. Kuo HM, Lin CY, Lam HC, et al. PTEN overexpression attenuates angiogenic processes of endothelial cells by blockade of endothelin-1/endothelin B receptor signaling. Atherosclerosis 2012; 221: 341-9.

18. Collet G, Szade K, Nowak W, et al. Endothelial precursor cell-based therapy to target the pathologic angiogenesis and compensate tumor hypoxia. Cancer Lett 2016; 370: 345-57.

19. Kieda C, El Hafny-Rahbi B, Collet G, et al. Stable tumor vessel normalization with $\mathrm{pO}(2)$ increase and endothelial PTEN activation by inositol trispyrophosphate brings novel tumor treatment. J Mol Med (Berl) 2013; 91: 883-99.

20. Cantelmo AR, Conradi LC, Brajic A, et al. Inhibition of the Glycolytic Activator PFKFB3 in Endothelium Induces Tumor Vessel Normalization, Impairs Metastasis, and Improves Chemotherapy. Cancer Cell 2016; 30: 968-85.

21. Teng MW, Ngiow SF, Ribas A, Smyth MJ. Classifying Cancers Based on T-cell Infiltration and PD-L1. Cancer Res 2015; 75: 2139-45.

22. Bielawska-Pohl A, Crola C, Caignard A, Gaudin C, Dus D, Kieda C, Chouaib S. Human NK cells lyse organ-specific endothelial cells: analysis of adhesion and cytotoxic mechanisms. J Immunol 2005; 174: 5573-82.

23. Bielawska-Pohl A, Blesson S, Benlalam H, et al. The anti-angiogenic activity of IL-12 is increased in iNOS-/- mice and involves NK cells. J Mol Med (Berl) 2010; 88: 775-84.

24. Dondero A, Casu B, Bellora F, et al. NK cells and multiple myeloma-associated endothelial cells: molecular interactions and influence of IL-27. Oncotarget 2017; 8: 35088-102.

25. Hansen W1, Hutzler M, Abel S, et al. Neuropilin 1 deficiency on CD4+Foxp3+ regulatory T cells impairs mouse melanoma growth. J Exp Med 2012; 209: 2001-16.

26. Grabie N1, Gotsman I, DaCosta R,et al. Endothelial programmed death-1 ligand 1 (PD-L1) regulates CD8+ T-cell mediated injury in the heart. Circulation 2007; 116: 2062-71. 
27. Rodig N, Ryan T, Allen JA, et al. Endothelial expression of PD-L1 and PD-L2 down-regulates CD8+ T cell activation and cytolysis. Eur J Immunol 2003; 33: 3117-26.

28. Zhao L, Li C, Liu F, et al. A blockade of PD-L1 produced antitumor and antimetastatic effects in an orthotopic mouse pancreatic cancer model via the PI3K/Akt/mTOR signaling pathway. Onco Targets Ther 2017; 10: 2115-26.

29. Song M, Chen D, Lu B, et al. PTEN loss increases PD-L1 protein expression and affects the correlation between PD-L1 expression and clinical parameters in colorectal cancer. PLoS One 2013; 8: e65821.

30. Giuliani M, Janji B, Berchem G. Activation of NK cells and disruption of PD-L1/PD-1 axis: two different ways for lenalidomide to block myeloma progression. Oncotarget 2017; 8: 24031-44.

31. Parsa AT, Waldron JS, Panner A, et al. Loss of tumor suppressor PTEN function increases B7-H1 expression and immunoresistance in glioma. Nat Med 2007; 13: 84-8.

32. Schreiber RD, Old LJ, Smyth MJ. Cancer immunoediting: integrating immunity's roles in cancer suppression and promotion. Science 2011; 331: 1565-70.

33. Garrido F, Algarra I, García-Lora AM. The escape of cancer from T lymphocytes: immunoselection of MHC class I loss variants harboring structural-irreversible "hard" lesions. Cancer Immunol Immunother 2010; 59: 1601-6.

34. Bedognetti D, Hendrickx W, Ceccarelli M, Miller LD, Seliger B. Dis entangling the relationship between tumor genetic programs and immune responsiveness. Curr Opin Immunol 2016; 39: 150-8.

35. Gabrilovich DI, Ostrand-Rosenberg S, Bronte V. Coordinated regulation of myeloid cells by tumours. Nat Rev Immunol 2012; 12: 253-68.

36. Kanterman J, Sade-Feldman M, Baniyash M. New insights into chronic inflammation-induced immunosuppression. Semin Cancer Biol 2012; 22: 307-318.

37. Schito L, Semenza GL. Hypoxia-inducible factors: master regula tors of cancer progression. Trends Cancer 2016; 2: 758-770.

38. Borsig L, Wolf MJ, Roblek M, Lorentzen A, Heikenwalder M. Inflammatory chemokines and metastasis - tracing the accessory. Oncogene 2014; 33: 3217-3224

39. De Sanctis F, Solito S, Ugel S, Molon B, Bronte V, Marigo I. MDSCs in cancer: Conceiving new prognostic and therapeutic targets. Biochim Biophys Acta 2016; 1865: 35-48.

40. Parker KH, Beury DW, Ostrand-Rosenberg S. Myeloid-derived suppressor cells: critical cells driving immune suppression in the tumor microenvironment. Adv Cancer Res 2015; 128: 95-139.

41. Kumar V, Patel S, Tcyganov E, Gabrilovich DI1.The nature of myeloid-derived suppressor cells in the tumor microenvironment. Trends Immunol 2016; 37: 208-20.

42. Meirow Y, Kanterman J, Baniyash M. Paving the Road to Tumo Development and Spreading: Myeloid-Derived Suppressor Cells are Ruling the Fate. Front Immunol 2015; 6: 523.

43. Ostrand-Rosenberg S. Myeloid-derived suppressor cells: more mechanisms for inhibiting antitumor immunity. Cancer Immuno Immunother 2010; 59: 1593-600.

44. Gabrilovich DI, Nagaraj S. Myeloid-derived suppressor cells as regulators of the immune system. Nat Rev Immunol. 2009; 9: 162-74

45. Bronte V, Brandau S, Chen SH, et al. Recommendations for myeloid-derived suppressor cell nomenclature and characterization standards. Nat Commun 2016; 7: 12150.

46. Solito S, Marigo I, Pinton L, Damuzzo V, Mandruzzato S, Bronte V. Myeloid-derived suppressor cell heterogeneity in human cancers. Ann N Y Acad Sci 2014: 1319: 47-65.

47. Filipazzi P, Huber V, Rivoltini L. Phenotype, function and clinical implications of myeloid-derived suppressor cells in cancer patients. Cancer Immunol Immunother 2012; 61: 255-63.

48. Umansky V, Sevko A. Melanoma-induced immunosuppression and its neutralization. Semin Cancer Biol 2012; 22: 319-26.

49. Umansky V, Blattner C, Gebhardt C, Utikal J. CCR5 in recruitment and activation of myeloid-derived suppressor cells in melanoma. Cancer Immunol Immunother 2017; 66: 1015-23.

50. Condamine T, Gabrilovich DI. Molecular mechanisms regulating myeloid-derived suppressor cell differentiation and function. Trends Immunol 2011; 32: 19-25.
51. Yu H, Pardoll D, Jove R. STATs in cancer inflammation and immunity: a leading role for STAT3. Nat Rev Cancer 2009; 9: 798-809.

52. Sade-Feldman M, Kanterman J, Ish-Shalom E, Elnekave M, Horwitz E, Baniyash M. Tumor necrosis factor-alpha blocks differentiation and enhances suppressive activity of immature myeloid cells during chronic inflammation. Immunity 2013; 38: 541-54.

53. Kumar V, Gabrilovich DI. Hypoxia-inducible factors in regulation of immune responses in tumour microenvironment. Immunology 2014; 143: 512-9.

54. Noman MZ, Chouaib S. Targeting hypoxia at the forefront of anticancer immune responses. Oncoimmunology 2014; 3: e954463.

55. Corzo CA, Condamine T, Lu L, et al. HIF-1alpha regulates function and differentiation of myeloid-derived suppressor cells in the tumor microenvironment. J Exp Med 2010; 207: 2439-53.

56. Noman MZ, Janji B, Hu S, Wu JC, Martelli F, Bronte V, Chouaib S. Tumor-promoting effects of myeloid-derived suppressor cells are potentiated by hypoxia-induced expression of miR-210. Cancer Res 2015; 75: 3771-87

57. Bogdan C. Nitric oxide and the immune response. Nat Immunol 2001; 2: 907-16.

58. Molon B, Ugel S, Del Pozzo F, et al. Chemokine nitration prevents intratumoral infiltration of antigen-specific T cells. J Exp Med 2011; 208: 1949-62.

59. Umansky V, Schirrmacher V. Nitric oxide-induced apoptosis in tumor cells. Adv. Cancer Res 2001; 82: 107-31.

60. Raber P1, Ochoa AC, Rodríguez PC. Metabolism of L-arginine by myeloid-derived suppressor cells in cancer: mechanisms of T cell suppression and therapeutic perspectives. Immunol Invest 2012; 41: 614-34

61. Noman MZ, Desantis G, Janji B, Hasmim M, Karray S, Dessen P, Bronte V, Chouaib S. PD-L1 is a novel direct target of HIF-1alpha, and its blockade under hypoxia enhanced MDSC-mediated T cell activation. J Exp Med 2014; 211: 781-90.

62. Ishida Y, Agata Y, Shibahara K, Honjo T. Induced expression of PD-1, a novel member of the immunoglobulin gene superfamily, upon programmed cell death. EMBO J 1992; 11: 3887-95.

63. Boussiotis VA, Chatterjee P, Li L. Biochemical signaling of PD-1 on T cells and its functional implications. Cancer J 2014; 20: 265-71.

64. Gajewski TF, Woo SR, Zha Y, Spaapen R, Zheng Y, Corrales L Spranger S. Cancer immunotherapy strategies based on overcoming barriers within the tumor microenvironment. Curr Opin Immunol 2013; 25: 268-76

65. Spranger S, Spaapen RM, Zha Y, Williams J, Meng Y, Ha TT, Gajewski TF. Up-regulation of PD-L1, IDO, and T(regs) in the melanoma tumor microenvironment is driven by CD8(+) T cells. Sci Transl Med 2013; 5: 200ra116.

66. Sceneay J, Parker BS, Smyth MJ, Möller A. Hypoxia-driven immunosuppression contributes to the pre-metastatic niche. Oncoimmunology 2013; 2: e22355.

67. Hong J, Tobin NP, Rundqvist H, et al. Role of tumor pericytes in the recruitment of myeloid-derived suppressor cells. J Natl Cancer Inst 2015; 107: pii: djv209

68. Chiu DK, Xu IM, Lai RK, et al. Hypoxia induces myeloid-derived suppressor cell recruitment to hepatocellular carcinoma through chemokine (C-C motif) ligand 26. Hepatology 2016; 64: 797-813.

69. Sitkovsky MV, Hatfield S, Abbott R, Belikoff B, Lukashev D, Ohta A. Hostile, hypoxia-A2-adenosinergic tumor biology as the next barrier to overcome for tumor immunologists. Cancer Immunol Res 2014; 2: 598-605.

70. Allard B, Beavis PA, Darcy PK, Stagg J. Immunosuppressive activities of adenosine in cancer. Curr Opin Pharmacol 2016; 29: 7-16.

71. Whiteside TL, Mandapathil M, Schuler P. The role of the adenosinergic pathway in immunosuppression mediated by human regulatory T cells (Treg). Curr Med Chem 2011; 18: 5217-23.

72. Umansky V, Shevchenko I, Bazhin AV, Utikal J. Extracellular adenosine metabolism in immune cells in melanoma. Cancer Immunol Immunother 2014; 63: 1073-80.

73. Junger WG. Immune cell regulation by autocrine purinergic signalling. Nat Rev Immunol 2011; 11: 201-12.

74. Wang L, Fan J, Thompson LF, Zhang Y, Shin T, Curiel TJ, Zhang B. CD73 has distinct roles in nonhematopoietic and hematopoietic cells to promote tumor growth in mice. J. Clin Invest 2011; 121: 2371-82. 
75. Beavis PA, Stagg J, Darcy PK, Smyth MJ. CD73: a potent suppressor of antitumor immune responses. Trends Immunol 2012; 33: 231-7.

76. Stagg J, Beavis PA, Divisekera U, Liu MC, Möller A, Darcy PK, Smyth MJ. CD73-deficient mice are resistant to carcinogenesis. Cancer Res 2012; 72: 2190-6.

77. Ryzhov S, Novitskiy SV, Goldstein AE, Biktasova A, Blackburn MR, Biaggioni I, Dikov MM, Feoktistov I. Adenosinergic regulation of the expansion and immunosuppressive activity of CD11b+Gr1+ cells. J Immunol 2011; 187: 6120-9.

78. Ohta A, Sitkovsky M. Extracellular adenosine-mediated modulation of regulatory T cells. Front Immunol 2014; 5: 304.

79. Sitkovsky MV. T regulatory cells: hypoxia-adenosinergic suppression and re-direction of the immune response. Trends Immunol 2009; 30: 102-8.

80. Noman MZ, Buart S, Van Pelt J, et al. The cooperative induction of hypoxia-inducible factor-1 alpha and STAT3 during hypoxia induced an impairment of tumor susceptibility to CTL-mediated cell Iysis. J Immunol 2009; 182: 3510-21.

81. Noman MZ, Janji B, Kaminska B, et al. Blocking hypoxia-induced autophagy in tumors restores cytotoxic T-cell activity and promotes regression. Cancer Res 2011; 71: 5976-86.

82. Noman MZ, Buart S, Romero P, Ketari S, Janji B, Mari B, Mami-Chouaib F, Chouaib S. Hypoxia-inducible miR-210 regulates the susceptibility of tumor cells to lysis by cytotoxic T cells. Cancer Res 2012; 72: 4629-41.

83. Janji B, Viry E, Moussay E, et al. The multifaceted role of autophagy in tumor evasion from immune surveillance. Oncotarget 2016; 7 : 17591-607.

84. Baginska J, Viry E, Berchem G, et al. Granzyme B degradation by autophagy decreases tumor cell susceptibility to natural killer-mediated lysis under hypoxia. Proc Natl Acad Sci U S A 2013; 110: 17450-5.

85. Messai Y, Noman MZ, Hasmim M, et al. ITPR1 protects renal cancer cells against natural killer cells by inducing autophagy. Cancer Res 2014; 74: 6820-32.

\section{Address for correspondence}

\section{Salem Chouaib}

NSERM

Gustave Roussy

Univ. Paris-Sud

Universite Paris-Saclay

Villejuif, France

e-mail: Salem.chouaib@igr.fr 